\title{
Stufendiagnostik - massgeschneiderte und kosteneffektive Laboranalytik
}

\author{
Philipp Walter, dipl. pharm., Medizinisches Labor Olten AG
}

Medizinische Fachlaboratorien bieten Tests mit hervorragenden Sensitivitäts- und Spezifitätsparametern in einem breitgefächerten Angebot an. Die Vielfalt der verfügbaren Parameter verleitet leicht zur Annahme, dass eine breitangewendete Labordiagnostik die ärztliche Praxis in jedem Fall am besten unterstützt. Der vorliegende Artikel zeigt auf, dass der Einsatz einer breiten Palette von Laborparametern, die zuweilen in umfassenden Analysenprofilen angeboten werden, nicht in jedem Fall der effektivste Weg ist. Tatsächlich bringen unnötige Untersuchungen oft nicht nur keinen Informationsgewinn, sondern können Anlass zu weiteren überflüssigen und kostspieligen klinischen Untersuchungen geben. In Zusammenarbeit mit Ihrem Fachlabor können Sie verschiedene Laborparameter dagegen differenziert einsetzen, um in einem stufendiagnostischen Prozess die Aussage jedes Messergebnisses optimal zu nutzen. Innovative Laboratorien bieten der Arztpraxis die Möglichkeit, ein individuell vordefiniertes stufenweises Vorgehen ohne zusätzlichen administrativen Aufwand zu nutzen.

\section{Prinzip der Stufendiagnostik}

Grundsätzlich bieten sich in vielen Fragestellungen Parameter mit orientierendem Charakter an. Diese werden in der Stufendiagnostik verwendet, um in einem ersten Schritt Auskunft darüber zu erlangen, ob mit weiteren Tests überhaupt zusätzliche Informationen mit klinisch-diagnostischer Relevanz gewonnen werden können. Die Stufendiagnostik basiert auf der Erarbeitung der klinischen Prätestwahrscheinlichkeit in der ärztlichen Praxis. Zusammen mit den Kenntnissen des Fachlabors über entsprechende statistische Charakteristika eines Tests kann eine differenzierte Aussage über Sinn und Unsinn zusätzlicher Tests erarbeitet und eine kostenintensive «Schrotschussdiagnostik» vermieden werden. Das Prinzip der Stufendiagnostik bezieht sich dabei nicht ausschliesslich auf die Verordnung von Laboranalysen, sondern auf die gezielte Einbettung von Laboranalysen in sämtliche klinisch-diagnostische Aktivitäten in der Arztpraxis.

\section{Diagnostischer Wert von Laboranalysen}

Der diagnostische Wert von Laboranalysen hängt stark von der Anamnese in der Arztpraxis ab. Die Abschätzung der Prätestwahrscheinlichkeit, d.h. die Ermittlung des Erkrankungsrisikos aufgrund der anamnestischen Erhebung vor der Bestimmung der Laborwerte, ist in vielen Fällen Voraussetzung für eine sinnvolle Interpretation der Laborergebnisse. Grundsätzlich ist dabei eine mittlere Wahrscheinlichkeit für das Vorliegen einer bestimmten Erkrankung hinsichtlich des diagnostischen Wertes einer verordneten Laboranalyse optimal - das Differenzierungsvermögen (basierend auf den Sensitivitäts- und Spezifitätsparametern) eines Testes kommt dabei am stärksten zum Tragen.

Basierend auf Prätestwahrscheinlichkeit und Testresultat ergibt sich die Prätestwahrscheinlichkeit für Folgeuntersuchungen. Hierbei entscheidet sich, ob die Bestimmung weiterer Parameter Sinn macht. In bestimmten Fällen wird der Informationszuwachs durch Folgetests so gering sein, dass deren Messung vom diagnostischen Standpunkt her keine Vorteile bringen kann.

\section{Praxis der Stufendiagnostik}

Mit Ihrem Fachlabor können Sie - individuell auf die Bedürfnisse Ihrer Praxis ausgerichtet Algorithmen definieren, nach welchen die Stufendiagnostik der von Ihnen entsprechend gekennzeichneten Aufträge abgearbeitet wird. Medizinische Laboratorien, welche die Stufendiagnostik als Mittel zum sinnvollen Einsatz der finanziellen Ressourcen im Gesundheitswesen unterstützen, bieten diese Möglichkeit auf dem Auftragsformular an. Damit können Sie dem Fachlabor die Kompetenz überlassen, im Rahmen der getroffenen Vereinbarung die verfügbare Testpalette sinnvoll einzusetzen. Die Stärken der Fachlabors zur Optimierung der diagnostischen Anforderung durch die Arztpraxis kommen damit ohne zeitlichen und administrativen Mehraufwand zur Geltung. Zudem kann es in bestimmten Fällen sinnvoll sein, Stufendiagnostik 
durch Nachverordnungen zu betreiben. Das medizinische Labor gewährt dazu die fachgerechte Lagerung des eingesandten Probenmaterials.

\section{Beispiele für die Praxis}

Ein stufenweises Vorgehen bewährt sich beispielsweise in der Schilddrüsendiagnostik, bei der Bestimmung des Lipidstoffwechsels, bei der Abklärung von Hepatitiden, beim Screening nach Prostatakarzinomen sowie bei der Differenzierung von Proteinurien.

\section{Seminare 2005}

\section{Praxiscomputerworkshop}

\section{Teilnehmende}

Der Workshop richtet sich an praxiseröffnende sowie an bereits praxistätige Ärztinnen und Ärzte.

\section{Inhalt}

Die Seminarteilnehmer/innen erhalten die Gelegenheit, die Praxisadministrationssoftware von sechs EDV-Unternehmen kennenzulernen. Sie erhalten einen Einblick in TARMED-kompatible Lösungen für die Leistungsabrechnung, die Führung elektronischer Krankengeschichten, die Einbindung von Laborgeräten und von Finanzbuchhaltungslösungen. Bei den anwesenden Firmen handelt es sich jeweils um fünf Hersteller von EDV-Gesamtlösungen in der Arztpraxis sowie um eine Abrechnungskasse. Zudem wird innerhalb des Einführungsreferates zum Thema «TrustCenter - was habe ich in meiner Praxis davon?» der Sinn und Zweck der TrustCenter sowie deren Nutzen für die einzelne Arztpraxis aufgezeigt.

\section{Ziel}

Die Workshopteilnehmer/innen erhalten einen ersten Überblick über führende Softwarelösungen. Sie erfahren, wie bei der Evaluation eines Ärztepaketes zweckmässigerweise vorgegangen wird und welche Fehler vermieden werden sollten.
Verschiedene Vertreter der daCapo-Gruppierung (www.dacapo.ch) werden in loser Folge praxisorientiert über stufendiagnostisches Vorgehen bei der Verordnung von Laboranalysen informieren. In den kommenden Monaten werden dabei Anämieabklärungen, Autoimmunerkrankungen und die Thrombophiliediagnostik exemplarisch erörtert.

daCapo: med. chem. Labor Olten, Labor Prof. Krech Kreuzlingen, Mikrogen Zürich, Labor Dr. Rothen Basel, Medics Labor Bern.

\section{Kosten}

Der Workshop ist für FMH-Mitglieder gratis.

\section{Daten}

Nr. 15 Donnerstag, 24. November 2005 KongressZürich 14.00-17.30 Uhr haus

\section{Folgende Softwareanbieter können Sie kennenlernen}

Ärztekasse, Urdorf (MediWin CB Pro)

Als Marktleader seit 1964 im Bereich Praxisadministration können Sie bei der Ärztekasse auf eine ganzheitliche Lösung für Ihre Fragen betreffend Abrechnungsvarianten, Computerwahl, Netzwerke, Formular- und Briefbearbeitung etc. zählen. Die statistischen Erhebungen (im Auftrag der FMH, Roko) sind ein anerkanntes betriebswirtschaftliches und standesorganisatorisches Hilfsmittel für die moderne Praxisführung. Nebst innovativen Ideen und Lösungen sowie Kooperationspartnerschaft mit 10 TrustCentern ist uns eine umfassende Kundenbetreuung ein Anliegen. Unsere Standorte befinden sich in Basel, Bern, Chur, Crissier, Genf, Le Landeron, Lugano, Luzern, St. Gallen, Thônex und Zürich. Sie definieren Ihre Wünsche - wir bieten Ihnen die Lösung! 
Delemed AG, Gümligen (delemed ${ }^{\circledR}$ PEX II)

Delemed AG entwickelt und vertreibt seit 14 Jahren erfolgreich PEX II, die einfache, sympathische und effiziente Software für die Arztpraxis und Kliniken. Durch einen modularen Aufbau kann das System jederzeit den aktuellen Bedürfnissen des Kunden angepasst werden. Dank unserer Vielseitigkeit im medizinischen Umfeld und unserem Kundenstamm von über 620 Anwendern sind wir ein optimaler Partner für Sie. Unsere Kunden übermitteln bereits über ein Jahr ihre Rechnungen erfolgreich an die Trustcenter.

Kern Concept, Gossau (Aeskulap)

Aeskulap ist ein extrem schnelles Windows-Programm, das zusammen mit einer SQL-Datenbank höchste Verarbeitungsgeschwindigkeit, Kompaktheit und schnellste Zugriffsmöglichkeiten gewährleistet. Aeskulap bietet für alle Bedürfnisse eine optimale Lösung: vom einfachen Abrechnungssystem mit sämtlichen Leistungserfassungsmöglichkeiten bis zur vollelektronischen, papierlosen KG-Führung. Aeskulap ist ein modular aufgebautes, anpassbares System mit einer Vielzahl von Modulen. Als einziges Softwarehaus haben wir für den TARMED einen TARMED-Validator sowie einen TARMED-Optimizer direkt in die Ärztesoftware integriert. Die sofortige Validierung und Optimierung nach jeder Positionseingabe gewährleistet vollständige Rechnungen, da technische Grundleistungen, Prozentzuschläge, Alterszuschläge vollautomatisch hinzugefügt werden, ohne dass dafür dutzendfache Leistungsblöcke notwendig sind.

\section{Triamun AG, Gümligen (Praxidata)}

Triamun AG ist eines der führenden Systemhäuser für Medizininformatik. Triamun steht für Sicherheit, Innovation und gute Lösungen, womit ein reibungsloser Ablauf im Praxisalltag gewährleistet ist. Mit den Praxidata-Modulen wurde eine wirtschaftliche Komplettlösung geschaffen, die sich den unterschiedlichen Bedürfnissen in den Arztpraxen optimal anpasst. Mit der Neuentwicklung der Produktpalette PraxiCenter $^{\circledR}$ auf Microsoft.NET-Technologie hat Triamun für ihre Kunden wiederum eine gute Lösung gefunden. Damit setzt das Unternehmen jenseits herkömmlicher 32bit-Lösungen neue Massstäbe und sichert dadurch die Zukunft seiner Kunden. Triamun AG hat anhand ihrer 12jährigen Erfahrung auf diesem Gebiet eine neue, einfache, übersichtliche und hochstrukturierte elektronische Krankengeschichte entwickelt (Patient View ${ }^{\circledR}$ ). Triamun ist die erste
Firma in der Schweiz, die eine echte ASP-Lösung für die Arztpraxis anbieten kann.

\section{Praxinova AG, Kaltenbach / Stein am Rhein (Praxistar)}

Praxinova AG betreut seit über zwanzig Jahren ambulante Arztpraxen rund um die EDV. Mit grossem Erfolg. Entschieden sich doch innert zwei Jahren über 200 Arztpraxen für das neue Abrechnungsprogramm Praxistar. Unter Einsatz modernster Entwicklungstechnologie und der Zusammenarbeit mit ETH-juniors leitete Praxistar die neue Entwicklungsqualität ein. Das innovative und sehr übersichtliche Praxistar etablierte sich in kürzester Zeit bei Ärzten, die sich Praxistar zeigen liessen und es vergleichen konnten. Durch den modulartigen Programmaufbau kann Praxistar auf die individuellen Bedürfnisse der Arztpraxis ausgelegt werden. Alle Fachrichtungen finden hier «ihre» Lösung. Praxistar mit dem einzigartigen TARMED-Assistenten reduziert den Arbeitsaufwand auf das absolute Minimum. Der gesamte EDV-Bereich der Arztpraxis wird mit weiteren Produkten (elektronische Krankengeschichte KAGE, elektronische Agenda praxiTIME) von Praxinova vollständig und mit höchster Betriebssicherheit abgedeckt. Durch eine enge und persönliche Kundenbindung wird die getätigte Investition in die Zukunft mit motivierten Mitarbeitern abgerundet. Vergleichen Sie!

\section{Vitodata AG, Ohringen $b$. Winterthur \\ (vitoMed Administrationssystem)}

Die Vitodata AG feiert in diesem Jahr das 25jährige Bestehen. Die Unternehmung konzentriert sich auf Praxis- und Kliniklösungen. Die innovative Haltung eröffnet laufend neue Einsatzgebiete - immer mit dem entsprechenden Nutzen für die Anwender. In den ersten 25 Jahren stand die Abrechnung mit der EDV im Vordergrund. Heute ist die Vitodata AG auch bei der elektronischen Krankengeschichtenführung an der Spitze im Schweizer Markt. Die Marktführerschaft ist für das ganze Team der Vitodata AG eine Verpflichtung, im Sinne des Investitionsschutzes für die Kunden den Fortbestand zu sichern und unternehmerisch und ethisch stets korrekt $\mathrm{zu}$ handeln.

\section{Anmeldung}

FMH Consulting Services, Simone Köpfli, Burghöhe 1, 6208 Oberkirch, Tel. 04192500 77, Fax 0419210586 oder via Internet unter www. fmhservices.ch. 